\title{
Planar Printed Shorted Monopole Antenna with Coupled Feed for LTE/WWAN Mobile Handset Applications
}

\author{
Do-Gu Kang and Youngje Sung \\ Department of Electronic Engineering, Kyonggi University Yeongtong-gu, Suwon-si, Gyeonggi-do, Republic of Korea \\ Correspondence should be addressed to Youngje Sung; yjsung@kgu.ac.kr
}

Received 28 June 2012; Accepted 6 December 2012

Academic Editor: Antonio Faraone

Copyright (c) 2013 D.-G. Kang and Y. Sung. This is an open access article distributed under the Creative Commons Attribution License, which permits unrestricted use, distribution, and reproduction in any medium, provided the original work is properly cited.

A shorted monopole antenna with coupled feed for LTE/WWAN mobile handset applications is described. The basic resonance of the shorted monopole combines with the resonance formed by the coupling between the coupling strip and the feeding pad to cover the LTE700, GSM850, and GSM900 bands. Both the feeding pad and the coupling strip operate with the shorting strip as a loop antenna. The resonance of the loop antenna and the harmonics of the shorted monopole combine to cover the GSM1800, GSM1900, UMTS, and LTE2300 bands. A stable and omnidirectional radiation pattern with reasonable gain has been observed over the operating bandwidth.

\section{Introduction}

Technological advancements and the diversity of needs in wireless communication are leading to the development of antennas for smaller, lighter, and more multifunctional mobile handsets [1]. Consequently, planar monopoles [24] enabling pentaband WWAN (wireless wide area network) operation have been developed. The WWAN band covers the GSM850 (824-894 MHz), GSM900 (880-960 MHz), GSM1800 (1710-1880 MHz), GSM1900 (1850-1990 MHz), and UMTS (1920-2170 MHz) bands. These developed planar monopoles are, respectively, a monopole with coupled strips [2], a CPW-fed LI-shaped monopole [3], and a coupledfed shorted monopole [4]. Recently, coupled-fed shorted monopoles covering not only the WWAN band but also the LTE band have been developed. The LTE band covers LTE700 (698-798 MHz), LTE2300 (2300-2400 MHz), and LTE2500 (2500-2690 MHz) [5, 6]. These monopoles operating in the WWAN/LTE bands are, respectively, a monopole with a tuning stub [5] and a monopole with a chip inductorloaded shorted strip [6].

In this paper, a shorted monopole antenna with coupled feed for LTE/WWAN bands is proposed. The base resonance of the shorted monopole combines with the resonance formed by the coupling between the feeding pad and the coupling strip to include the LTE700, GSM850, and GSM900 bands. The loop antenna is formed by both the coupled feed structure and the shorting strip. The loop resonance and a harmonic element combine to cover the GSM1800, GSM1900, UMTS, and LTE2300 bands. Therefore, the proposed structure covers the LTE700, LTE2300, GSM850, GSM900, GSM1800, GSM1900, and UMTS bands.

\section{Antenna Geometry}

Figure 1 shows the proposed antenna which consists of a long radiator $(\mathrm{AB})$, a coupling strip (BC), a feeding pad, and a shorting strip. $l_{1}$ denotes the total length of the long radiator. $l_{2}$ denotes the length of the part generating the coupled feed, and $g$ denotes the gap between the feeding pad and the coupling strip; these are the two parameters that affect the coupled feed. $l_{3}$ denotes the length of the feeding pad. The antenna covers an area of $18 \times 49 \mathrm{~mm}^{2}$ on the upper surface of an FR4 substrate with a $0.6 \mathrm{~mm}$ thick. The total area of the substrate is $118 \times 49 \mathrm{~mm}^{2}$; the ground (GND) found on the lower surface of the substrate covers an area of $100 \times 49 \mathrm{~mm}^{2}$.

\section{Simulated and Measured Results}

Figure 2 shows the simulated and measured reflection coefficients for the proposed antenna and reference 1 . The 


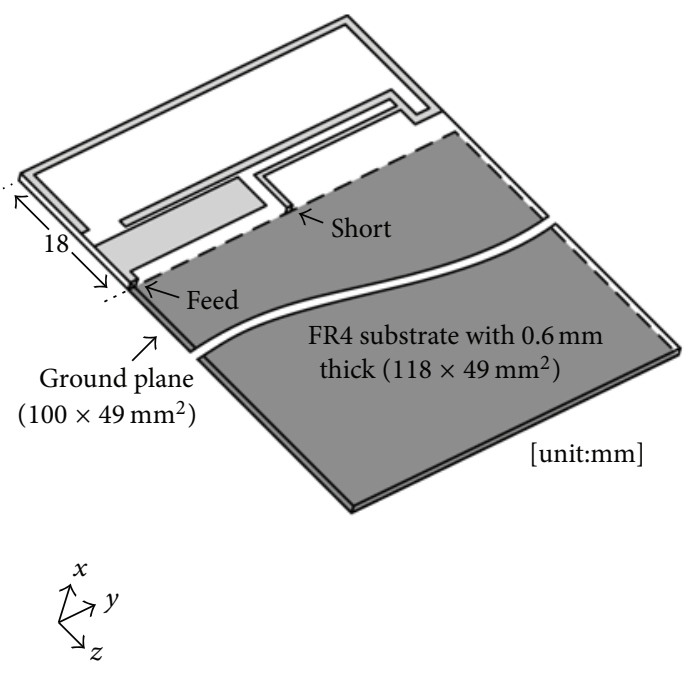

(a)

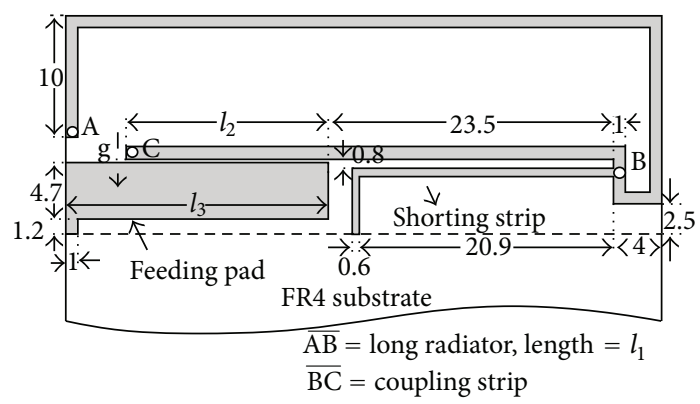

(b)

FIGURE 1: (a) 3D geometry of the proposed antenna. (b) The antenna geometry as seen from above.

parameters for the proposed antenna were set to $l_{1}=78 \mathrm{~mm}$, $l_{2}=16.5 \mathrm{~mm}, g=0.3 \mathrm{~mm}$, and $l_{3}=21.5 \mathrm{~mm}$. The prototype of the proposed antenna is based on the foregoing optimized parameters and is fabricated as shown in Figure 3. The simulation was carried out through HFSS. The simulated results were nearly identical to the measured results. The proposed antenna has four resonant frequencies $\left(f_{1}, f_{2}, f_{3}\right.$, and $f_{4}$ ). The low band covers the LTE700, GSM850, and GSM900 bands, and the high band covers the GSM1800, GSM1900, UMTS, and LTE2300 bands. All the bands operated properly with a $-6 \mathrm{~dB}$ bandwidth which is a widely used value for internal WWAN antennas in practical mobile phone application.

If the proposed antenna's result is compared to reference 1 's (the proposed antenna with direct feed) result, the effect of the coupled feed can be observed. The matching characteristics of the base resonance were improved by the coupled feed that increases the electrical length. Therefore, the resonance frequency decreased. The resonance combined with the second resonance that was formed by the coupled feed. The two resonances form dual resonance. Therefore, the proposed antenna achieves a low wideband through the coupled feed.

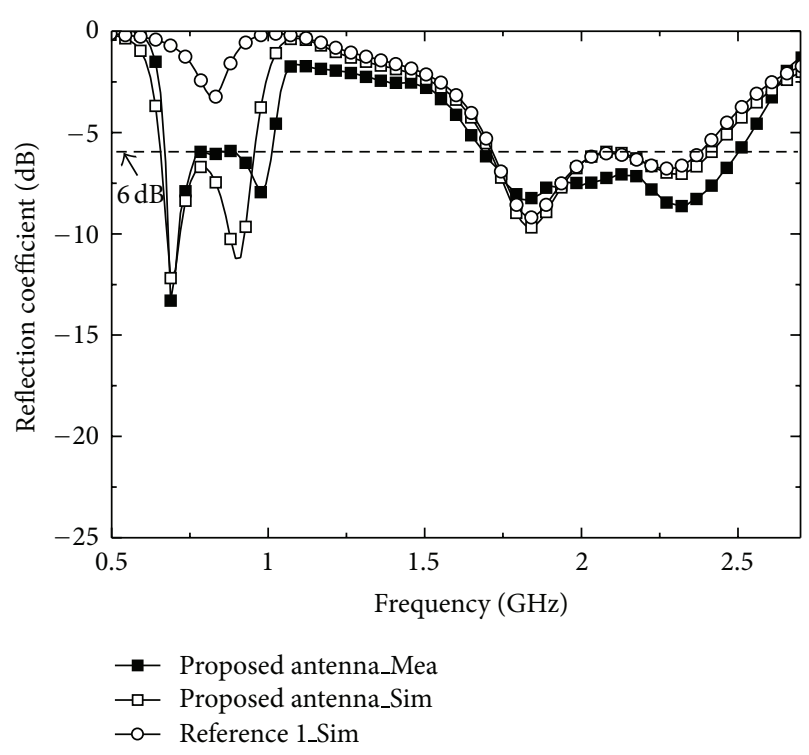

(a)

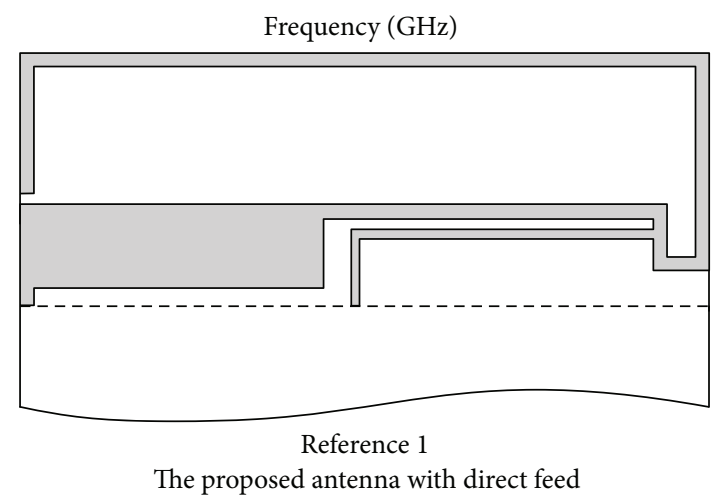

(b)

FIgURE 2: Simulated and measured reflection coefficients for the proposed antenna and reference 1.

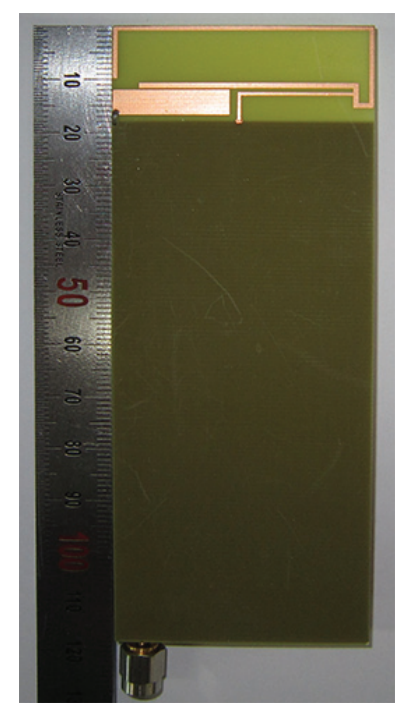

FIGURE 3: Fabricated prototype of the proposed antenna. 


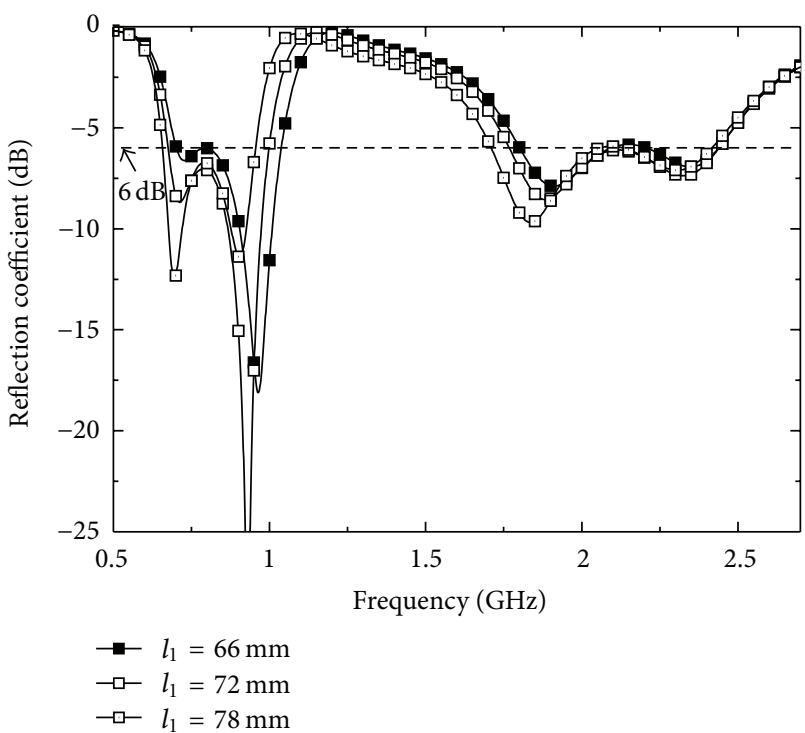

Figure 4: Simulated reflection coefficients relative to changes in the total length of the long radiator $l_{1}$.

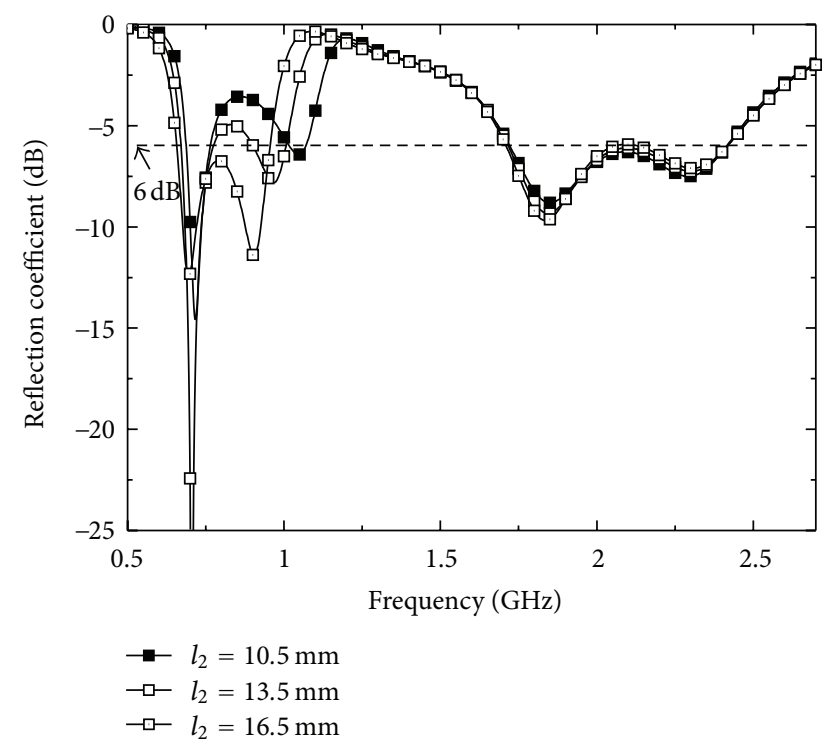

FIGURE 5: Simulated reflection coefficients relative to changes in the length of the part generating the coupled feed $l_{2}$.

Figure 4 shows the simulated reflection coefficients relative to the changes in the total length $l_{1}$ of the long radiator. The base resonance $f_{1}$ is formed by the long radiator's length which was a resonance length of $\lambda / 4$ at $f_{1}(0.69 \mathrm{GHz})$. Further, $f_{3}$ decreased, which proves that $f_{3}$ is a harmonic of $f_{1}$. As $l_{1}$ increased, the lower edge of the high band decreased from 1.8 to $1.71 \mathrm{GHz}$. Thus, the antenna covers the GSM1800 band. Therefore, considering the high band, $l_{1}$ was selected as $78 \mathrm{~mm}$.

Figure 5 shows the simulated reflection coefficients relative to the changes in length $l_{2}$ of the part generating the coupled feed $l_{2}$. Because $l_{2}$ affects the coupled feed, the

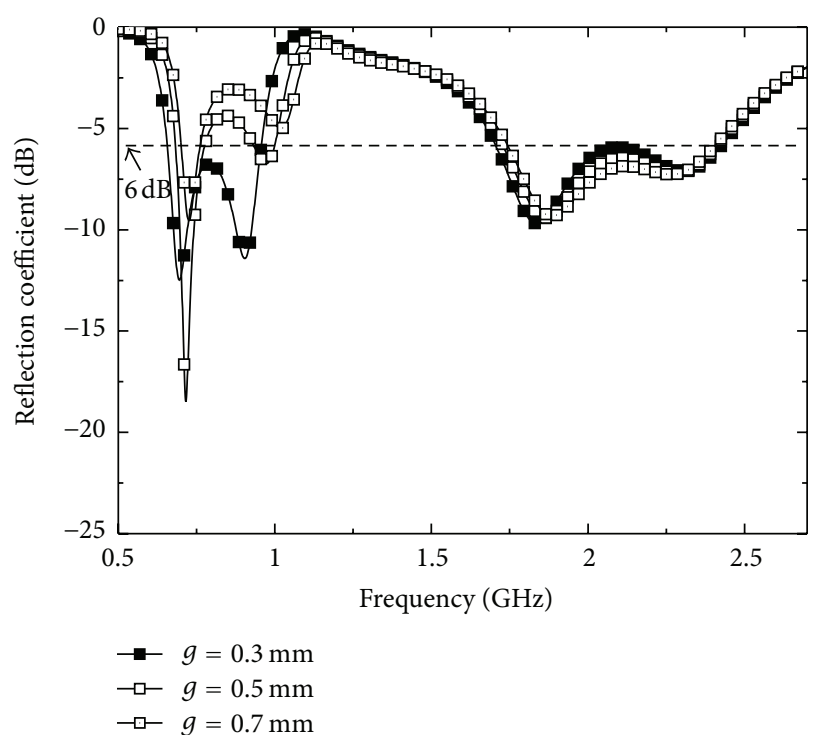

FIGURE 6: Simulated reflection coefficients relative to changes in the gap between feeding pad and coupling strip $g$.

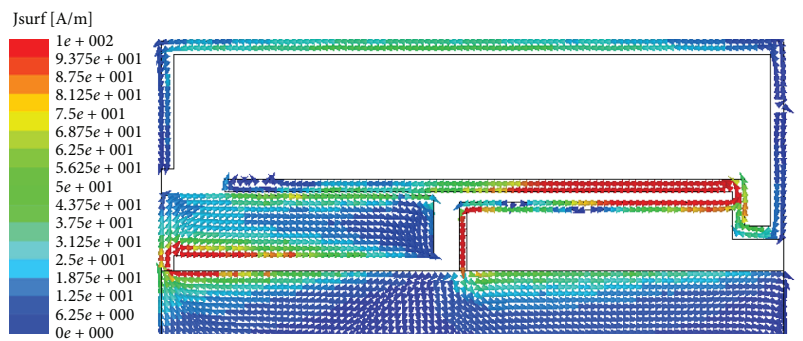

FIGURE 7: Surface current distribution simulated on the proposed antenna's radiator and GND at $f_{4}=2.3 \mathrm{GHz}$.

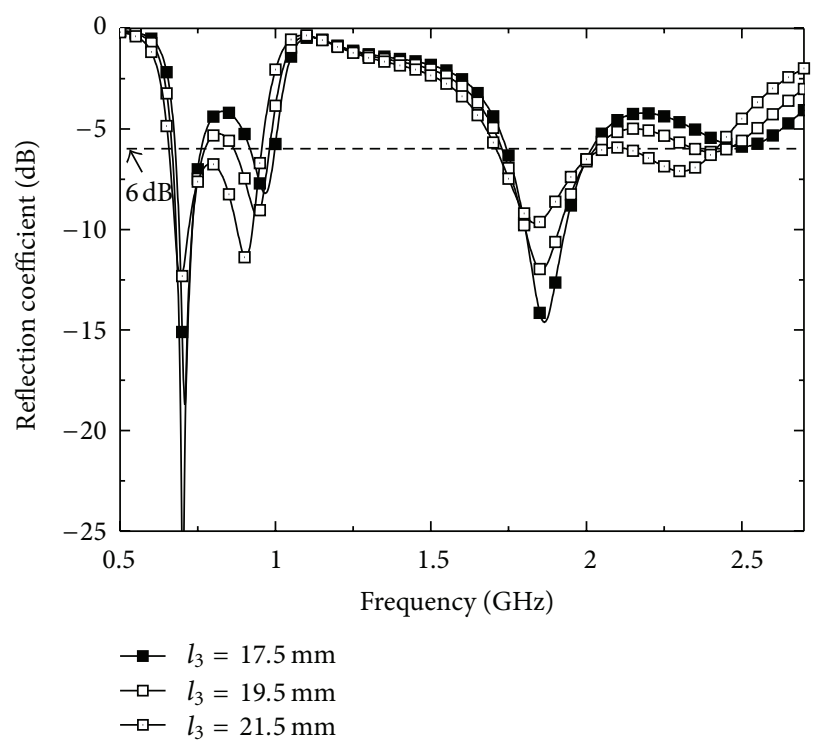

FIGURE 8: Simulated reflection coefficients relative to changes in the length of the feeding pad $l_{3}$. 


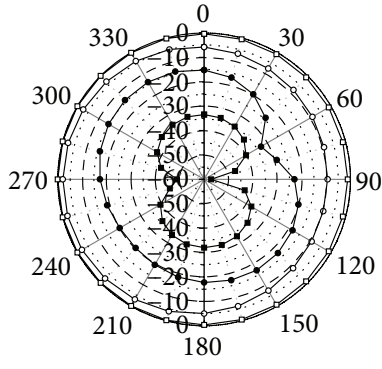

$x-y$ plane

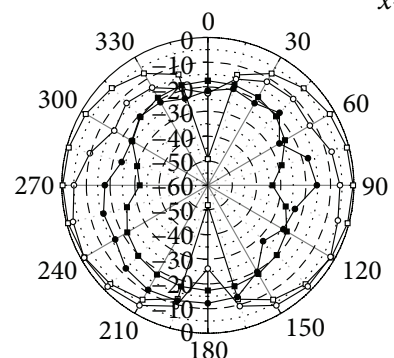

$\neg-E_{\theta}$ (simulated)

$\multimap E_{\theta}$ (measured)

$\rightarrow E_{\phi}$ (simulated)

$\rightarrow E_{\phi}$ (measured)

$x-z$ plane

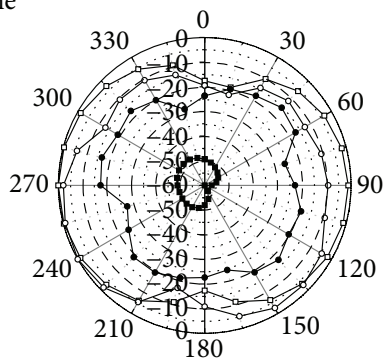

$\rightarrow-E_{\theta}$ (simulated)

$\multimap E_{\theta}$ (measured)

- $E_{\phi}$ (simulated)

$\longrightarrow E_{\phi}$ (measured)

$y$ - $z$ plane

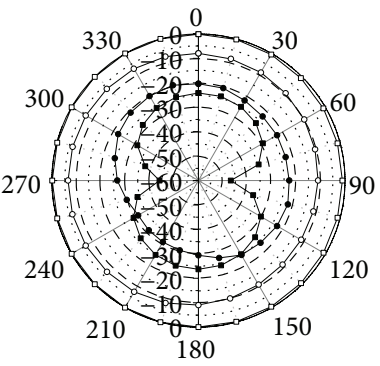

$x-y$ plane

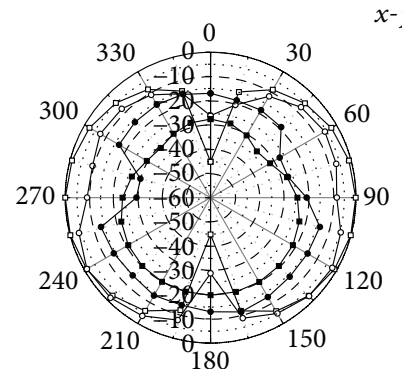

$\rightarrow-E_{\theta}$ (simulated)

$\multimap E_{\theta}$ (measured)

- $E_{\phi}$ (simulated)

$\longrightarrow E_{\phi}$ (measured)

$x$ - $z$ plane

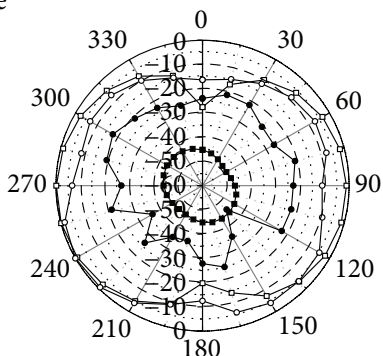

$\neg-E_{\theta}$ (simulated)

$\multimap E_{\theta}$ (measured)

$-E_{\phi}$ (simulated)

$\rightarrow E_{\phi}$ (measured)

$y-z$ plane

(a)

(b)

FIGURE 9: Simulated and measured 2D radiation patterns of proposed antenna: (a) $f_{1}=0.69 \mathrm{GHz}$, (b) $f_{2}=0.9 \mathrm{GHz}$.

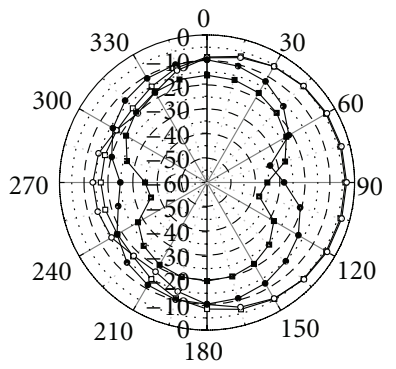

$x-y$ plane

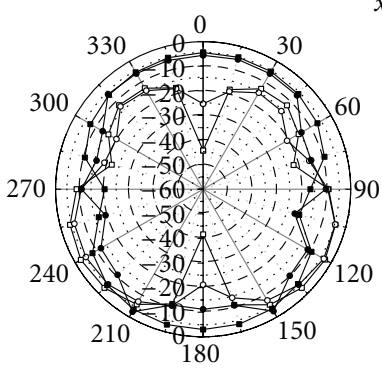

$\rightarrow-E_{\theta}$ (simulated)

$\multimap E_{\theta}$ (measured)

$\rightarrow E_{\phi}$ (simulated)

$\longrightarrow E_{\phi}$ (measured)

$$
x-z \text { plane }
$$

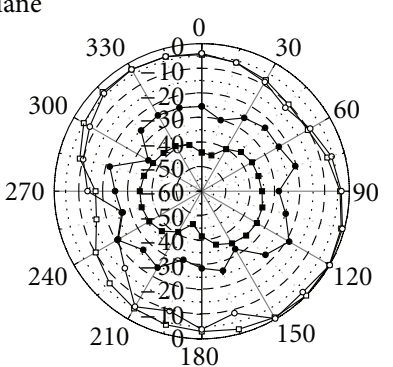

$\rightarrow \quad E_{\theta}$ (simulated)

$\multimap E_{\theta}$ (measured)

$\rightarrow E_{\phi}$ (simulated)

- $E_{\phi}$ (measured)

$$
y \text { - } z \text { plane }
$$

(a)
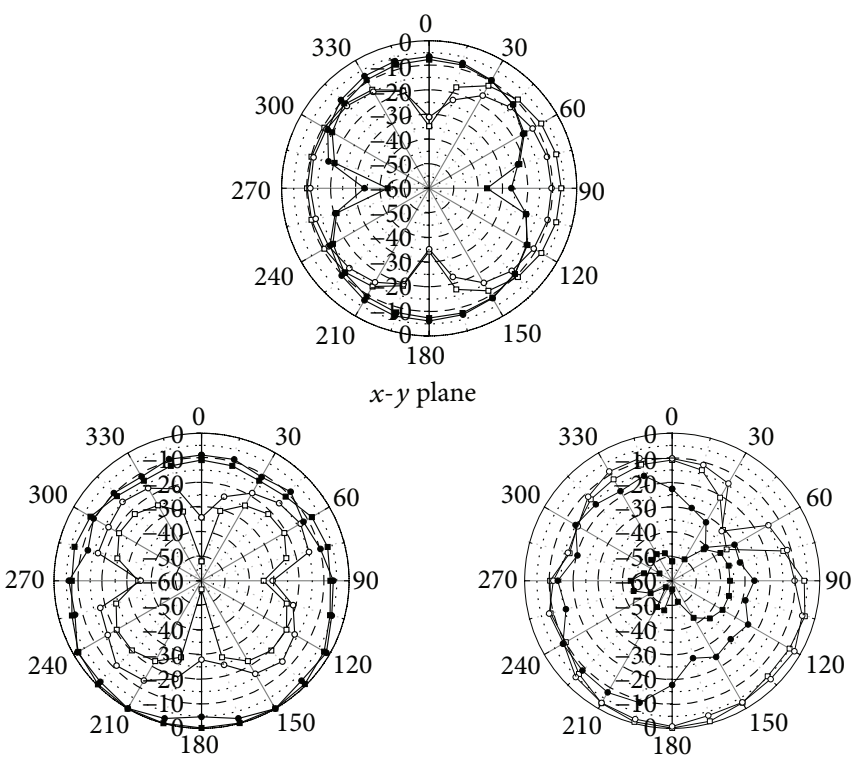

$\neg-E_{\theta}$ (simulated)

$\multimap E_{\theta}$ (measured)

$\rightarrow E_{\phi}$ (simulated)

$\longrightarrow E_{\phi}$ (measured)

$x$ - $z$ plane
$\rightarrow-E_{\theta}$ (simulated)

$\multimap E_{\theta}$ (measured)

$-E_{\phi}$ (simulated)

$\longrightarrow E_{\phi}$ (measured)

$y$ - $z$ plane

(b)

FIGURE 10: Simulated and measured 2D radiation patterns of proposed antenna: (a) $f_{3}=1.82 \mathrm{GHz}$, (b) $f_{2}=2.3 \mathrm{GHz}$. 


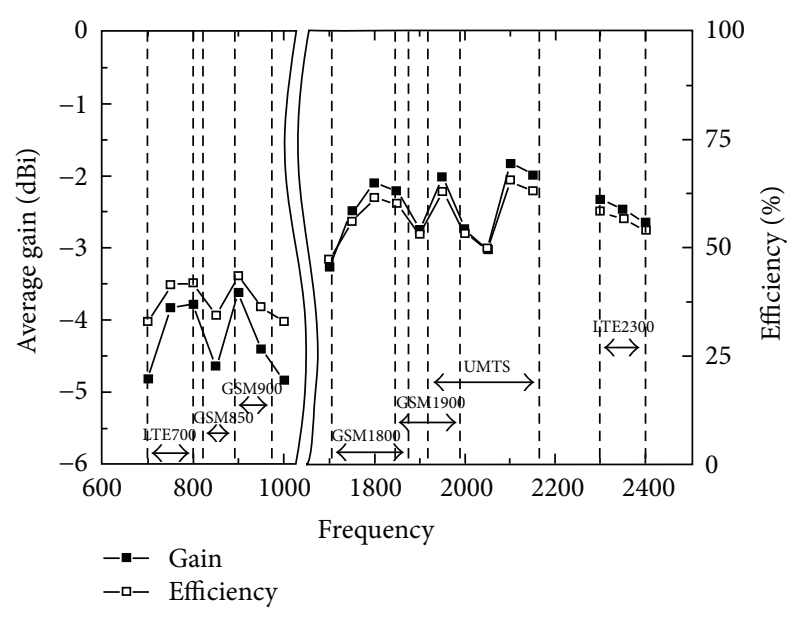

FIGURE 11: Measured average gain and efficiency of the proposed antenna.

resonance formed by the coupled feed was affected as $l_{2}$ changed. The base resonance formed by the long radiator further combined with the resonance formed by the coupled feed as $l_{2}$ increased. $l_{2}$ was selected as $16.5 \mathrm{~mm}$ to allow the antenna to have low wideband characteristics.

Figure 6 shows the simulated reflection coefficients relative to the changes in gap $g$ between the feeding pad and the coupling strip. As $g$ decreased, the matching of $f_{2}$ improved in the state the matching between $f_{1}$ and $f_{2}$ improved. Thus, considering the low band, $g$ was selected as $0.3 \mathrm{~mm}$.

Figure 7 shows the surface current distribution simulated on the proposed antenna's radiator and GND at $f_{4}=$ $2.3 \mathrm{GHz}$. The red area indicates a strong field, whereas the blue area indicates a weak field. The maximum current is located at the beginning of the feeding pad, and the null of the current is located at the end. The coupled gap was electrically connected. The maximum current on the coupling strip generated the maximum current on the shorting strip's end. The null is located between the maximum current on the shorting strip's end and the maximum current on the shorting strip's beginning. Through these findings, the path from the feeding pad to the coupled gap was combined with the path from the coupling strip to the shorting strip, which was the path of the loop operating at the $\lambda$ resonant length. Therefore, $f_{4}$ was the resonant frequency of the loop antenna.

Figure 8 shows the simulated reflection coefficients relative to the changes in length $l_{3}$ of the feeding pad. As $l_{3}$ increased, the matching characteristics between $f_{1}$ and $f_{2}$ improved with a decrease of $f_{2}$. Because $l_{3}$ affected the length of the coupled feed, its effect was analogous to the effect of $l_{2}$ and $g$ in the low band. Further, the matching characteristics between $f_{3}$ and $f_{4}$ improved with a decrease of $f_{4}$. To take into consideration the effect of both the high band and low band, $l_{3}$ was set to $21.5 \mathrm{~mm}$. When a parameter study of $l_{3}$ was compared with other parameter studies, it showed that $f_{4}$ was relatively more transferred by $l_{3}$. This is because $l_{3}$ resides in the loop path.

Figures 9 and 10 show the simulated and measured 2D radiation patterns of the proposed antenna at $f_{1}=0.69 \mathrm{GHz}$, $f_{2}=0.9 \mathrm{GHz}, f_{3}=1.82 \mathrm{GHz}$, and $f_{4}=2.3 \mathrm{GHz}$. The radiation pattern was measured on the $x-y, x-z$, and $y$ - $z$ planes, on the basis of the direction of the antenna placement shown in Figure 1(a). The measured co-pols closely matched the simulated co-pols; the measured cross-pols were higher than the simulated cross-pols. The reason for this is that the GND was extended by the cabling used to measure the radiation pattern. Dipole-like radiation patterns with omnidirectional radiation in the azimuthal plane ( $x-y$ plane) are observed at two resonances of the low band in Figure 9. Conversely, more nulls and changes occurred in the radiation pattern observed for the two high band resonances in Figure 10. The obtained radiation patterns were analogous to the radiation patterns of WWAN mobile phone antennas [7, 8]. In addition, stable radiation patterns were obtained in both the low band and the high band. Figure 11 shows the measured average gain and efficiency of the proposed antenna. At the LTE700/ GSM850/GSM900 band, the average gain varies from -4.8 to $-3.6 \mathrm{dBi}$. At the GSM1800/GSM1900/UMTS band, it varies from -3.2 to $-1.8 \mathrm{dBi}$, while it varies from -2.6 to $-2.3 \mathrm{dBi}$ at the LTE2300 band.

\section{Conclusions}

In this work, a shorted monopole antenna with a coupled feed for LTE/WWAN mobile handset applications was proposed. The base resonance of the shorted monopole and the resonance formed by the coupled feed structure combine to form a low wideband. The coupled feed structure and the shorting strip generate the loop antenna. The loop resonance combines with the harmonic element, which forms a high wideband. Therefore, the proposed structure is suitable for seven-band mobile handset applications, covering the LTE700, GSM850, GSM900, GSM1800, GSM1900, UMTS, and LTE2300 bands. Good radiation characteristics for frequencies over the operating bands were observed.

\section{Acknowledgment}

This research was supported by Basic Science Research Program through the National Research Foundation of Korea (NRF) funded by the Ministry of Education, Science, and Technology (2010-0021222).

\section{References}

[1] P. Ciais, R. Staraj, G. Kossiavas, and C. Luxey, "Design of an internal quad-band antenna for mobile phones," IEEE Microwave and Wireless Components Letters, vol. 14, no. 4, pp. 148-150, 2004.

[2] H. W. Liu and C. F. Yang, "Miniature multiband monopole antenna for WWAN operation in laptop computer," Electronics Letters, vol. 46, no. 1, pp. 21-23, 2010.

[3] Y. Jee and Y. M. Seo, "Triple-band CPW-fed compact monopole antennas for GSM/PCS/DCS/WCDMA applications," Electronics Letters, vol. 45, no. 9, pp. 446-448, 2009.

[4] C.-T. Lee and S.-W. Su, "Compact and printed, standalone wireless wide area network antenna incorporating a conductive wire at low cost," Microwave and Optical Technology Letters, vol. 54, no. 1, pp. 109-113, 2012. 
[5] S. C. Chen and K. L. Wong, "Small-size 11-band LTE/WWAN/ WLAN internal mobile phone antenna," Microwave and Optical Technology Letters, vol. 52, no. 11, pp. 2603-2608, 2010.

[6] S. C. Chen and K. L. Wong, "Wideband monopole antenna coupled with a chip-inductor-loaded shorted strip for LTE/WWAN mobile handset," Microwave and Optical Technology Letters, vol. 53, no. 6, pp. 1293-1298, 2011.

[7] K. L. Wong and C. H. Huang, "Compact multiband PIFA with a coupling feed for internal mobile phone antenna," Microwave and Optical Technology Letters, vol. 50, no. 10, pp. 2487-2491, 2008.

[8] B. N. Kim, S. O. Park, J. K. Oh, and G. Y. Koo, "Wideband built-in antenna with new crossed c-shaped coupling feed for future mobile phone application," IEEE Antennas and Wireless Propagation Letters, vol. 9, pp. 572-575, 2010. 

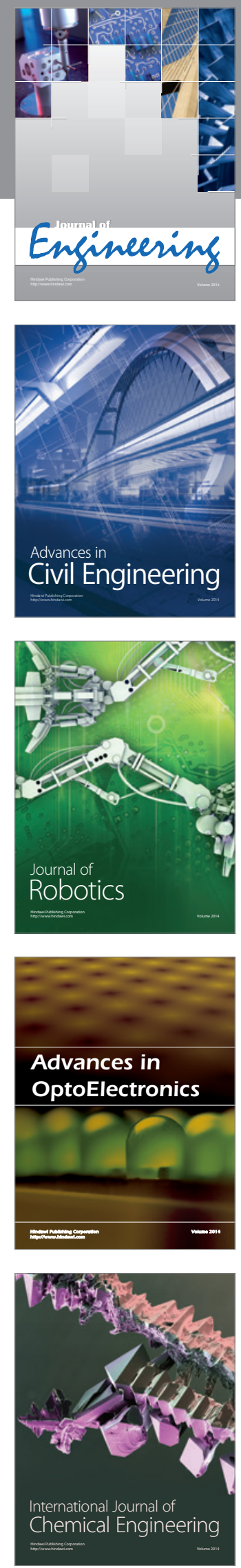

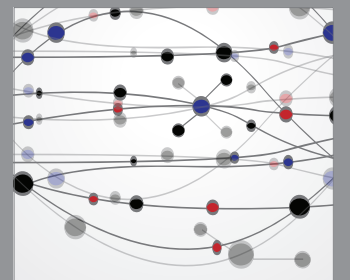

The Scientific World Journal
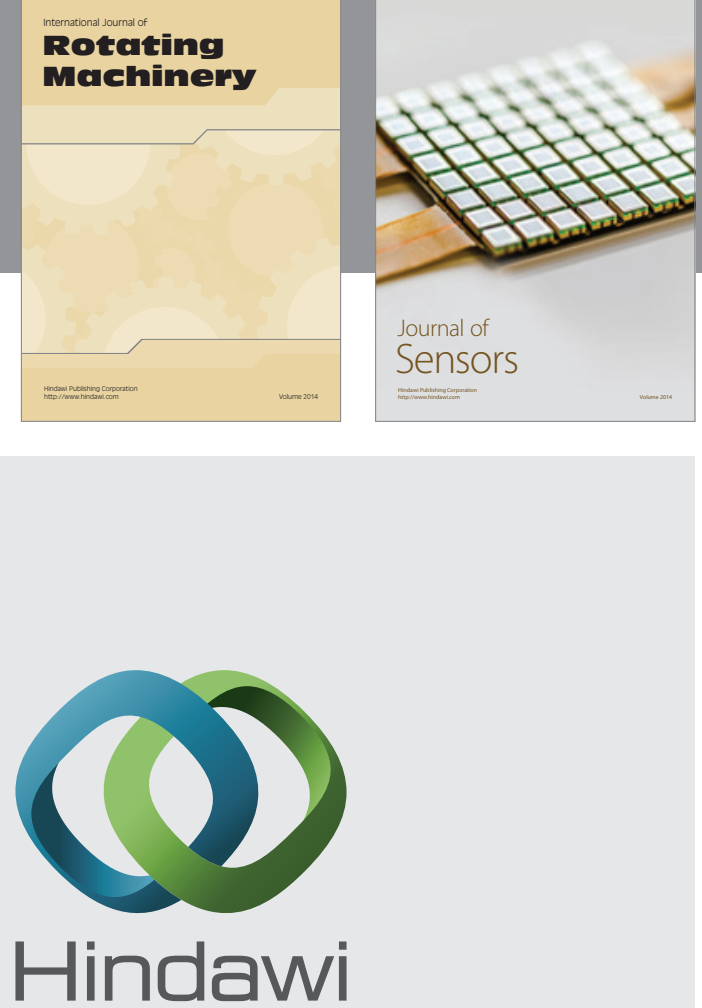

Submit your manuscripts at http://www.hindawi.com
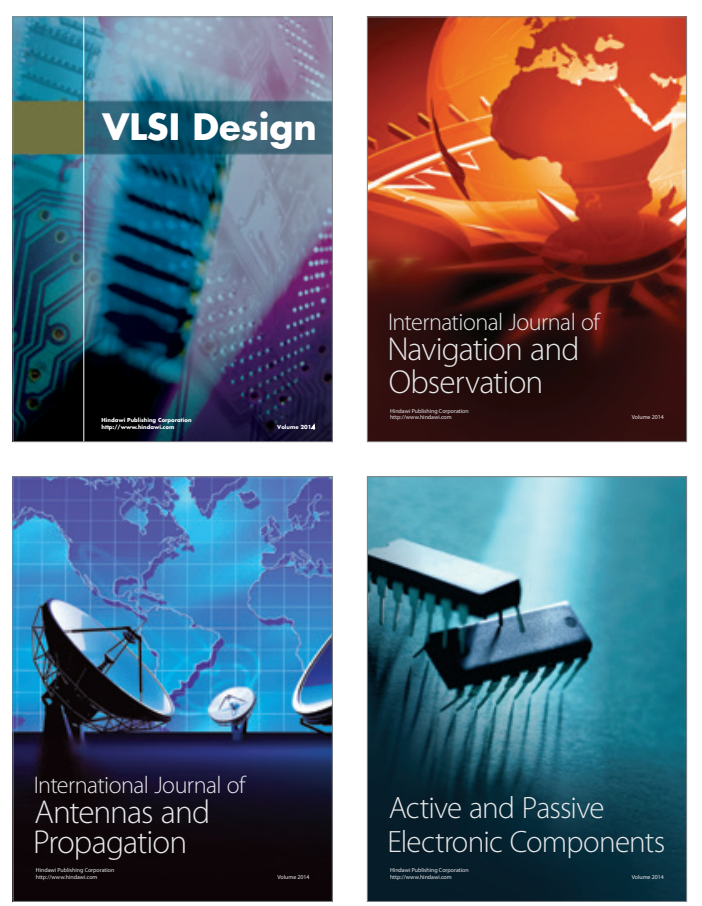
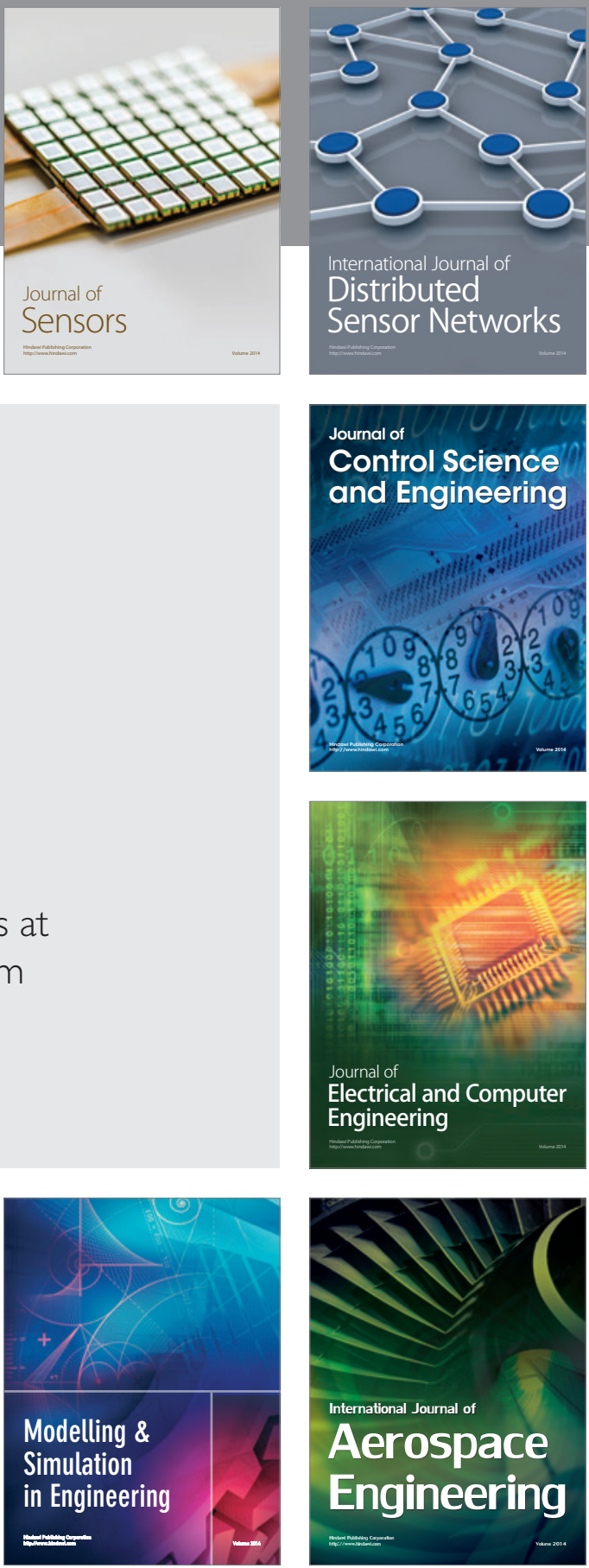

Journal of

Control Science

and Engineering
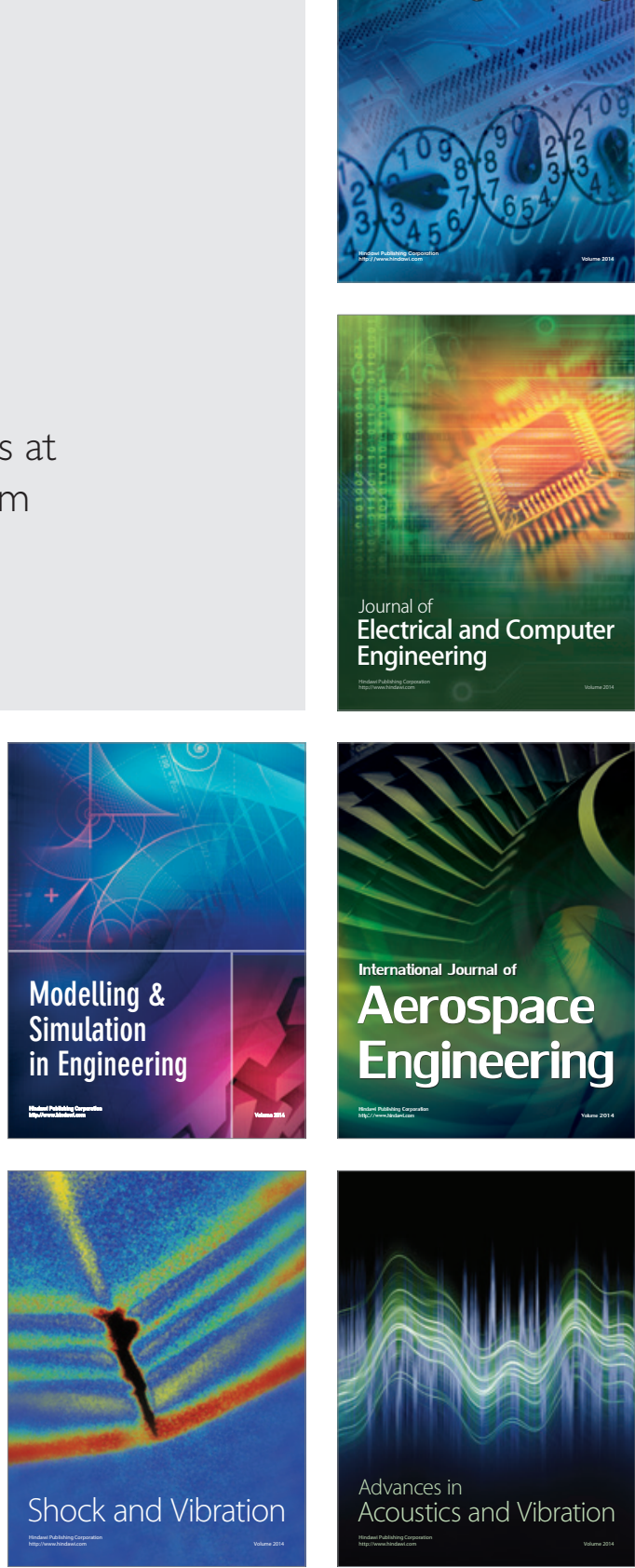